\title{
A DISTORTION THEOREM FOR DOUBLY CONNECTED REGIONS
}

\author{
MILTON N. PARNES ${ }^{1}$
}

ABSTRACT. In this paper we use a symmetrization result of Szegö and a geometric lemma to generalize a distortion theorem of Pblya and Szegö for simply connected regions to doubly connected regions.

1. Introduction. A set $S$ in the complex plane will be called $n$-fold symmetric about a point $p$ if every rotation of $2 \pi / n$ about $p$ maps $S$ onto itself. The following result is due to Pólya and Szegö [6].

Theorem A. Let $G$ be a convex region, $n$-fold symmetric about the origin 0 . For $p \in G$, we denote by $r(G, p)$ the inner conformal radius of $G$ with respect to $p$. Then

$$
r(G, 0) \geqq r(G, p)
$$

for every $p \in G$. Furthermore, equality is attained if and only if either $p=0$, or $G$ is an infinite strip and $p$ is equidistant from the two parallel boundary lines of $G$.

In this paper we will use a simple geometric lemma and a symmetrization result of Szegö [7] to prove the above result and to establish a generalization of this result to doubly connected regions. Throughout this paper, we assume, for convenience, that each component of the complement of any region has at least two boundary points.

Let $R$ be a doubly connected region (or ring), $E$ the bounded component of the complement of $R$ and $G$ the union of $R$ with $E$. We denote the ring $R$ by $R=(G, E)$, and the logarithmic capacity of $R$ by $\operatorname{Cap}(G, E)$. The set translation of $E$ by the complex number $b$ will be denoted, as usual, by $E+b$. We obtain the following

Theorem B. Let $R=(G, E)$ be a doubly connected region such that

(1) $G$ is a convex region $n$-fold symmetric about the origin 0 ,

Received by the editors October 10, 1969.

AMS subject classifications. Primary 3040; Secondary 5230.

Key words and phrases. Doubly connected regions, $n$-fold symmetric, convex sets, distortion theorem, extremal problem.

1 The results in this paper are contained in the author's doctoral dissertation at Wayne State University written under the direction of Professor W. Seidel. The author also wishes to acknowledge helpful discussions with Professors D. Waterman, T. Nishiura and J. Krzyz. 
(2) $E$ is starlike with respect to 0 and $m$-fold symmetric about 0 , and

(3) $m$ and $n$ have a common divisor $d \geqq 2$. Then for any $p \in G$ such that $E+p \subset G$, we have

$$
\operatorname{Cap}(G, E+p) \geqq \operatorname{Cap}(G, E) .
$$

Furthermore, equality is attained if and only if either $p=0$, or $G$ is an infinite strip and $p$ is equidistant from the two parallel boundary lines of $G$.

We also note that the infinite strip between two parallel lines is the only $n$-fold symmetric domain which is unbounded, and that which does not have a unique point about which it is symmetric. (Here, $n$ is equal to 2.) For these reasons, statements concerning this domain, although easy to prove and need only minor changes in the statements, are awkward and will not usually be stated or proved in this paper.

2. Szegö symmetrization theorem and geometric preliminaries. Let $S$ be a set in the complex plane, starlike with respect to $p$. We define the boundary function of $S$ with respect to $p$ by

$$
\phi(\theta)=\phi[S, p](\theta)=1 \text {.u.b. }\{|z-p|: z \in S, \arg (z-p)=\theta\},
$$

where $-\infty<\theta<\infty$ and $\phi[S, p]$ is allowed to be infinite. We also define the $n$-fold symmetrization function of $\phi[S, p]$ by

$$
\phi_{n}(\theta)=\phi[S, p]_{n}(\theta)=\left\{\prod_{j=0}^{n-1} \phi[S, p]\left(\theta+\frac{2 \pi j}{n}\right)\right\}^{1 / n} .
$$

A ring $(G, E)$ is said to be starlike with respect to $p$ if both $G$ and $E$ are starlike with respect to $p$. The following theorem is a summary of some results of Szegö [7].

THEOREM 2.1. Let $R=(G, E)$ be a ring starlike with respect to 0 . Suppose that $\phi$ and $\psi$ are the corresponding boundary functions of $G$ and $E$ with respect to $p$. Then for each integer $n \geqq 2$,

(1) $D\left(\phi_{n}\right)=\left\{\rho e^{i \theta}: 0 \leqq \rho<\phi_{n}(\theta)\right\}$ is open (possibly the whole plane);

(2) $D^{-}\left(\psi_{n}\right)=\left\{\rho e^{i \theta}: 0 \leqq \rho \leqq \psi_{n}(\theta)\right\}$ is compact (possibly the single point 0$)$;

(3) if $\phi_{n}$ is not identically $\infty$ and $\psi_{n}$ is not identically 0 , then $\left(D\left(\phi_{n}\right), D^{-}\left(\psi_{n}\right)\right)$ forms a ring;

(4) $r\left(D\left(\phi_{n}\right), 0\right) \geqq r(G, p)$; and

(5) if the conditions in (3) are given, $\operatorname{Cap}(G, E) \geqq \operatorname{Cap}\left(D\left(\phi_{n}\right), D^{-}\left(\psi_{n}\right)\right)$.

We call $D\left(\phi_{n}\right), D^{-}\left(\psi_{n}\right)$ and $\left(D\left(\phi_{n}\right), D^{-}\left(\psi_{n}\right)\right)$ the $n$-fold symmetrization of the open set $G$, the compact set $E$ and the ring $R=(G, E)$ re- 
spectively. The proofs of (1), (2), and (3) can be shown by using the semicontinuity properties of $\phi, \phi_{n}, \psi$, and $\psi_{n}$.

Definition. An $n$-star consists of a point $p$ called the vertex, and $n$ vectors emanating from $p$ equally spaced at an angle $2 \pi / n$. These vectors, called the rays of the $n$-star, are allowed to have infinite length. The length of an $n$-star $T$, denoted by $|T|$, is the sum of the lengths of its rays. An $n$-star will be called regular, if all its rays have equal length. Two $n$-stars $T$ and $P$ are said to be parallel if each ray of $T$ is parallel to a corresponding ray of $P$.

Lemma 2.2. Let $G$ be a regular $n$-gon in the complex plane with the origin 0 as center and with sides $s_{1}, \cdots, s_{n}$ labeled counterclockwise, such that $s_{1}$ is perpendicularly bisected by the positive real axis. Let $T$ be an $n$-star with vertex 0 and rays $a_{1}, \cdots, a_{n}$, such that $a_{j}$ terminates on the side $s_{j} ; j=1, \cdots, n$. Suppose $P$ is any $n$-star with vertex $p \in G$ and rays $b_{1}, \cdots, b_{n}$, such that each $b_{j}$ is parallel to $a_{j}$ and terminates on $s_{j}$. ( $s_{j}$ may have to be extended.) Then $|T|=|P|$.

Proof. Suppose that the rays of $T=T_{0}$ and $P=P_{0}$ are perpendicular to the sides of $G$. Form the triangles whose vertices are the point $p$ and two adjacent vertices of $G$. Then the sum of the areas of these triangles equals the area of $G$. That is, if $L$ denotes the length of a side $s_{j}$ of $G$, then

$$
\text { Area of } G=\sum_{i=1}^{n}\left|b_{i}\right| L / 2=\left|P_{0}\right| L / 2
$$

where $\left|b_{i}\right|$ is the length of $b_{i}$. Similarly,

$$
\text { Area of } G=\left|T_{0}\right| L / 2 \text {. }
$$

Hence, $\left|T_{0}\right|=\left|P_{0}\right|$. Now suppose that $T=T_{\theta}$ and $P=P_{\theta}$ are such that each ray forms an angle $\theta$ with the corresponding ray of $T_{0}$ and $P_{0}$. Then

$$
\left|T_{\theta}\right|=\left|T_{0}\right| \sec \theta=\left|P_{0}\right| \sec \theta=\left|P_{\theta}\right| .
$$

This completes the proof.

Lемма 2.3. Let $G$ be a convex region in the complex plane, $n$-fold symmetric with respect to the origin 0 , and let $T$ be an $n$-star with vertex 0 and rays terminated on the boundary of $G$. Suppose that $Q$ is another $n$-star, parallel to $T$, and with vertex $p \in G$ and rays terminated on the boundary of $G$. Then $|Q| \leqq|T|$.

Proof. If $G$ is unbounded, then $n=2$ and $G$ is an infinite strip. In this case, it is clear that $|Q|=|T|$. Now assume that $G$ is bounded. 
Label the points on the boundary of $G$ at which the rays of $T$ terminate $b_{1}, \cdots, b_{n}$ in a counterclockwise manner. Let $L_{1}$ be a line of support of $G$ at $b_{1}$ and $L_{j}, j=1, \cdots, n$, be the image of $L_{1}$ under a rotation of $2 \pi(j-1) / n$ about 0 . By the $n$-fold symmetry of $G, L_{j}$ is a line of support of $G$ at $b_{j}$. If $n=2, L_{1}$ and $L_{2}$ are parallel; and if $n>2, L_{1}, \cdots, L_{n}$ form a regular $n$-gon circumscribing $G$. Form the $n$-star $P$ with vertex $p$ and rays terminated at the (extended) sides of this regular $n$-gon such that $P, Q$ and $T$ are parallel. Clearly, $|Q| \leqq|P|$. If $n=2$, it is obvious that $|P|=|T|$ and if $n>2$, the previous lemma also gives $|P|=|T|$. Hence, $|Q| \leqq|T|$ as asserted.

3. Proofs of Theorems A and B. We need the following two theorems which we shall call the containment principles.

THEOREM 3.1. Let $(G, E)$ and $\left(G^{*}, E^{*}\right)$ be rings such that $G \subset G^{*}$ and $E^{*} \subset E$. Then

$$
\operatorname{Cap}\left(G^{*}, E^{*}\right) \leqq \operatorname{Cap}(G, E) .
$$

Equality holds if and only if $G=G^{*}$ and $E=E^{*}$.

THEOREM 3.2. Let $G$ and $G^{*}$ be simply connected domains with $G \subset G^{*}$ and let $p \in G$. Then

$$
r\left(G^{*}, p\right) \geqq r(G, p) .
$$

Equality holds if and only if $G=G^{*}$.

We shall now prove Theorems $\mathrm{A}$ and $\mathrm{B}$. Consider the ring $(G, E+p)$ in Theorem B, and assume this is the same $G$ and $p$ as in Theorem A. Let $\phi^{*}$ be the boundary function of $G$ with respect to $p$ and $\phi$ be that with respect to 0 . Let $\phi_{d}^{*}$ and $\phi_{d}$ be the corresponding $d$-fold symmetrizations of $\phi^{*}$ and $\phi$. Hence, by the arithmetic-geometric inequality and Lemma $2.3 \phi_{d}^{*}(\theta) \leqq \phi_{d}(\theta)=\phi(\theta)$ for all $\theta$, and it follows that $G=D\left(\phi_{d}\right) \supset D\left(\phi_{d}^{*}\right) \equiv G^{*}$. By the containment principles, we have $\operatorname{Cap}\left(G^{*}, E\right) \geqq \operatorname{Cap}(G, E)$ and $r\left(G^{*}, 0\right) \leqq r(G, 0)$.

By Theorem 2.1, $\operatorname{cap}(G, E+p) \geqq \operatorname{Cap}\left(G^{*}, E\right)$ and $r(G, p) \leqq r\left(G^{*}, 0\right)$. Combining these inequalities, we obtain $\operatorname{Cap}(G, E+p) \geqq \operatorname{Cap}(G, E)$ and $r(G, p) \leqq r(G, 0)$ as asserted in Theorems $\mathrm{A}$ and $\mathrm{B}$. The argument of when equalities hold follows easily by using the containment principles and by observing that, using the arithmetic-geometric inequality,

$$
\phi_{d}^{*}(\theta)=\phi_{d}(\theta) \quad(\text { for all } \theta)
$$

if and only if $G$ is $n$-fold symmetric about $p$.

As a corollary to Theorem $B$, we have 
Corollary 3.3. Let $B$ be a compact set starlike and $m$-fold symmetric with respect to $q$, and let $G$ be a region convex and $n$-fold symmetric about ihe origin 0 such that $G$ contains a set congruent to $B$. Consider the class of rings $R(G, E)$ where

(1) $G$ is as described above, $E$ is congruent to $B$;

(2) $m$ and $n$ have a common divisor $d \geqq 2$.

Then minimal capacity is attained in this class by a ring in which $q=0$.

Proof. By the continuity property of the logarithmic capacity and Theorem 4.1 in the following section, we know that there is a minimal ring in this class. The rest follows from Lemma 2.3 and Theorem B.

Observe that the corollary does not tell us the exact position of $E$ in $G$ other than $q=0$. When $G$ is an open disc or when $E$ is a closed disc, all positions (rotations) of $E$, where $q=0$, give the minimal capacity.

4. Some further comments. The following answers a question asked in a letter from Professor J. Pfaltzgraff. We shall perform a series of constructions in Theorem 4.2, which in conjunction with Lemma 4.1, imply that for every relatively prime pair $(m, n)$ there is an example which satisfies the conditions of Corollary 3.3 (except for condition 2) and for which the conclusion of Corollary 3.3 is false.

Of course this example shows Theorem B also cannot be changed in this way.

The following lemma is an immediate consequence of Teichmüller's Extremal Problem and the discussion in Künzi's book $[3$, p. 11].

Lemma 4.1. For every $M>0$ and $d>0$, there exists a $\delta(M, d)$ such that if $R=(G, E)$ is a ring such that

(1) The diameter of $E$ is greater thand.

(2) The distance from $E$ to the boundary of $G$ is less than $\delta(M, d)$. Then $\operatorname{Cap}(G, E) \leqq M$.

THEOREM 4.2. Let $G$ be a regular $n$-gon in the complex z-plane $(n=4 k-1,4 k, 4 k+1$ where $k=1,2, \cdots)$ with center at 0 . If $m$ is relatively prime to $n$, then there exists a regular $m$-star in $G$ whose length is strictly larger than any $m$-star with 0 as vertex.

Proof. Let $G$ be as in Lemma 2.2. In addition label the vertices of $G$ counterclockwise $V_{1}, \cdots, V_{n}$ where $V_{1}$, and $V_{n}$ determine $s_{1}$.

In the case $m=2$, our result is evident, since the line segment connecting $V_{(n+1) / 2}$ to $V_{1}$ does not pass through 0 , but has length larger than any 2 -star in $G$. For $m=2$ our result follows by continuity. 
If $m \geqq 3$ and $n=4 k-1,4 k, 4 k+1(K=1,2, \cdots)$, the proof is more complicated.

We determine the largest regular $m$-star which has 0 as vertex. Let $T_{0}$ be the $m$-star with 0 as vertex and rays that terminate on the boundary of $G$ and such that $\theta$ is the smallest positive angle that a ray of $T_{\theta}$ makes with the positive $x$-axis. For each $\theta$ the largest regular $m$-star with $\theta$ as vertex and parallel to $T_{\theta}$ has all its rays equal to the smallest ray of $T_{\theta}$.

Assume each apothem has length 1 . The shortest ray of $T_{0}$ is the ray perpendicular to $s_{1}$ and has length 1 . Since $m$ and $n$ are relatively prime, all the other rays of $T_{0}$ are not apothems and are therefore strictly larger. Letting $\theta$ increase, there is a first $\theta^{*}$ such that the smallest ray of $T_{\theta^{*}}$ is not unique. The following facts can be easily verified.

1. No three rays of any $T_{\theta}$ are equal. Therefore there are precisely two rays of $T_{\theta^{*}}$ which are of minimal length. One of these two rays terminates on $s_{1}$, and the other, call it $b$, on some other side, say $s_{h}$.

2. $s_{h}$ and $s_{1}$ are not parallel. (This is where we use the assumption $n=4 h$ and $n \neq 4 h+2$.) We can therefore draw lines $L_{1}$ and $L_{h}$ through the origin, parallel respectively to $s_{1}$ and $s_{h}$. This divides $G$ into four regions. Label these regions counterclockwise I, II, III, IV, where region $I$ lies to the right of the positive $y$-axis.

3 . If the argument of $b$ is greater than $\pi$ (less than $\pi$ ) by continuity there is a point $V$ in the region II (in the region III) such that the $m$-star $p$ parallel to $T_{\theta^{*}}$ with $V$ as vertex and rays that terminate on the boundary of $G$ will have its smallest ray a ray larger than $|\boldsymbol{b}|$.

Clearly there is a $m$-star with $V$ as vertex larger than any with 0 as vertex.

The example for the relatively prime pair $(m, n)$ is obtained from considering the regular $n$-gon, $G$ (if $n=4 k+2$, use the regular $2 n$-gon instead).

Let $E_{b}$ be an $m$-star contained in the interior of $G$ and larger than any $m$-star with 0 as vertex. The existence of $G$ and $E_{b}$ follows from 4.2. We may assume that the diameter of $E_{b}>1$. Let $\operatorname{Cap}\left(G, E_{b}\right)$ $=M$. Let $B^{*}$ be the largest regular $m$-star in $G$ as constructed in 4.2 and assume each of its rays has length $t$ and form $R=(G, B)$ where $B$ is the regular $m$-star with 0 as vertex, $B \subset B^{*}$, and rays of length $t-\delta(2 M, 1)$ where $\delta(2 M, 1)$ is defined in Lemma 4.1. It follows easily if $R=(G, E)$ is any ring with $G$ as above and $E$ congruent to $B$ with 0 as vertex, that $\operatorname{Cap} R \geqq 2 M$ while $\operatorname{Cap}\left(G, E_{b}\right)=M$. Let $E_{b}^{*}$ be congruent to $\mathrm{B}$ and such that $E_{b}^{*} \subset E_{b}$. Then by the containment 
principle $2 M=\operatorname{Cap}(R)>M \geqq \operatorname{Cap}\left(G, E_{b}^{*}\right)$. This concludes the construction and proves the claimed properties.

As pointed out by Pólya and Szegö, it is easy to show that we must assume $G$ to be convex. However, it might be interesting to drop the assumption of starlikeness on $E$. There is a generalization of Szegö's symmetrization due to Moshe Marcus [4] which does not depend on starlikeness to perform. However it does not have the property that it leaves nonstarlike $n$-fold symmetric sets unchanged.

Recently, C. K. Chui and the author [1] have discovered a partial converse to Lemma 2.3 as well as some other related results.

\section{REFERENCES}

1. C. K. Chui and M. N. Parnes, Measures of $N$-fold symmetry for convex sets, Proc. Amer. Math. Soc. (to appear).

2. G. H. Hardy, J. E. Littlewood and G. P6lya, Inequalities, Cambridge Univ. Press, New York, 1934.

3. H. P. Künzi, Quasikonforme Abbildungen, Ergebnisse der Mathematik und ihrer Grenzgebiete, Heft 26, Springer-Verlag, Berlin, 1960. MR 29 \#6013.

4. M. Marcus, Transformations of domains in the plane and applications in the theory of functions, Pacific J. Math. 14 (1964), 613-626. MR 29 \#2382.

5. M. N. Parnes, Symmetrization and conformal mapping, Dissertation, Wayne State University, Detroit, Mich., 1968.

6. G. P6lya and G. Szegö, Isoperimetric inequalities in mathematical physics, Ann. of Math. Studies, no. 27, Princeton Univ. Press, Princeton, N. J., 1951. MR 13, 270.

7. G. Szegö, On a certain kind of symmetrization and its applications, Ann. Mat. Pura Appl. (4) 40 (1955), 113-119. MR 17, 1074.

State University of New York at Buffalo, Amherst, New York 14226 